Rapid Reviews COVID-19

\title{
Review 2: "Comparison of
} infection control strategies to reduce COVID-19 outbreaks in homeless shelters in the United States: a simulation study"

Fiona Walsh ${ }^{1}$

${ }^{1}$ Principal Consultant, Mesurado Cooperative, USA

Published on: Nov 06, 2020

DOI: $10.1162 / 2 \mathrm{e} 3983 f 5 . b 68 a 5 b b 3$

License: Creative Commons Attribution 4.0 International License (CC-BY 4.0). 


\section{$\underline{\text { RR:C19 Evidence Scale rating by reviewer: }}$}

- Reliable. The main study claims are generally justified by its methods and data. The results and conclusions are likely to be similar to the hypothetical ideal study. There are some minor caveats or limitations, but they would/do not change the major claims of the study. The study provides sufficient strength of evidence on its own that its main claims should be considered actionable, with some room for future revision.

$* * * * * * * * * * * * * * * * * * * * * * * * * * * * * * * * * * * * * * *$

\section{Review:}

The preprint of "Comparison of infection control strategies to reduce COVID-19 outbreaks in homeless shelters in the United States: a simulation study" presents a microsimulation model of COVID-19 transmission in a representative homeless shelter over 30 days under simulated combinations of infection control strategies. Severe acute respiratory syndrome coronavirus 2 , the novel coronavirus that causes COVID19 , has had a disproportionate impact on people living in congregate settings, including nursing homes, correctional facilities, and homeless shelters. As noted in the preprint, this analysis is an important contribution to understanding which infection control strategies are the most effective in preventing COVID-19 transmission and minimizing outbreaks in homeless shelters-a population that has been historically underserved-to protect residents and staff.

The manuscript could be strengthened by addressing the following points:

First, the one-day turnaround time for PCR test results used in the model parameters is not realistic; most state and private laboratories have required more time (up to several days) for processing samples and resulting test-takers. Staff and residents would need to be isolated for more than one day while awaiting results. The model should account for a longer turnaround time for PCR test results, ideally informed by the current turnaround times for the state public health laboratories in the three selected settings (Boston, Seattle, and San Francisco).

Second, the preprint states that the initial population used in the modeling has the same composition of risk factors (age and co-morbidities) as the San Francisco shelter population. Since age and demographic characteristics determine whether an individual is treated as "low-risk" or "high-risk" in the model, a description of the 
demographic characteristics of the model population should be included in the manuscript. For example, in Supplementary Table 2, the probability of developing clinical symptoms for "low risk (age $<60$ years + no co-morbidities)" and "moderate risk (age $<60$ years + co-morbidities)" is the same (0.473), but individuals of any age with certain underlying medical conditions may have different time to presentation of fever and respiratory symptoms.

Third, individuals who have recovered from COVID-19 are assumed to remain immune in the microsimulation, but emerging research suggests that immunity may be timelimited. The possibility of reinfection is still not well-understood and so this point should be acknowledged in the description of the parameters.

Finally, the manuscript does not consider contact tracing to identify and quarantine exposed individuals within the model population, which would increase the number of individuals in isolation and also contribute to averting an outbreak. 